Lecture Note

\title{
A view about the nature and origin of the (elementary) electric charge and the possible size of the photon
}

\author{
C. D. Matsiras*
}

Physicist - Mathematician, 31 Kazazi str. 55133 Thessaloniki Hellas.

Received 16 April 2008; Accepted 23 February 2009

\begin{abstract}
The present study intends: on the one hand, to give an explanation of the origin and the nature of the elementary electric charge (e-), by determining the most possible reason why the electron shows Coulomb field, and by finding the mechanic equivalent to the electric charge; and on the other hand, to suggest a possible size for the photon by comparing maximum power wave lengths of photons and particles, taking into consideration the Planck length.

The initial idea arose from a previous paper of the author [1] entitled "Dwarf Primary Black Holes and Nucleons Indirect Theory of Unified Fields - Relation between Gravitational and Nuclear Potentials", where a new transformation formula of a collapsing mass and its reverse formula are introduced. From the application of the reverse formula, we conclude that electrons and nucleons (from quarks) were created from the crash of initial (primary) matter of the Universe, 9.11 millions and 1.674 billions of tons respectively, in the first infinitesimal flashes just after the Big Bang. Moreover, it is proved that: a) the nuclear forces of the nucleons come from the gravitational potential of their initial mass that is stored in them, b) the velocity of the collapse (crash) of a mass increases vertically, when its volume gets a critical value (critical volume), c) the gravity radius of the particle's initial mass is smaller or equal to the radius of the critical volume and the two radii take the same value, when the radius of the particle concurs with the gravity radius of the particle's mass and d) when the final velocity of collapse is equal to the velocity of light, then the collapsing mass changes into antimass (antimatter).
\end{abstract}

Keywords: Electron's electric charge, Coulomb potential, photon's size, Planck length.

\section{A View about the Nature and Origin of the (Elementary) Electric Charge.}

\subsection{Introduction}

As it is known, the electric charge is considered to be a principal concept in Physics, such as the mass, the length and the time. For these three concepts, however, we have direct and clear view, whereas as far as the electric charge is concerned, our view about its existence is based on the results of its action. The question raised is what the nature of the electric charge can be and how it originated. It is not matter, since the matter is a carrier of electric charge and more precisely, it carries electric charge in sums of an elementary quantity that comes from the electron's mass (positron).

In the next two sections, we will address, indirectly, these two subjects that constitute the concept of the electric charge by determining the most possible reason, why the electron shows Coulomb field and by finding the mechanic equivalent to the electric charge.

\subsection{Origin of electrons (positrons) - nucleons}

In a previous paper of the author [1], there is a view that the

\footnotetext{
* E-mail address: mmatsira@yahoo.gr

ISSN: 1791-2377 @ 2009 Kavala Institute of Technology. All rights reserved.
}

electrons come from crash (collapse) of waves of primary matter, most possibly of photons, and the nucleons, from Quarks, from crash of matter waves, maybe of leptons, in the first infinitesimal flashes, just after the Big-Bang. According to this view, the super atom, which most possibly was a photon (super photon), existed only for just a second and underwent immediately a tremendous and, inconceivable to the human brain, powerful explosion that powdered it in the several photons. The first infinitesimal flashes constitute the first period of the creation of the Universe, during which, according to Dr. S. Hawking's theory [2], we have quantum phenomena and Physics laws are unknown. In the next infinitesimal flashes $\left(10^{-43} \mathrm{sec}\right.$ and with temperature $10^{32}$ kelvin) we have the season of the Big Unification (Period of the First Crash according to the author). During this period, there is a crash of primary matter waves (possibly photons), with velocities of collapse almost equal to the velocity of light. The leptons are products of this crash, with the electrons-positrons being the most important representatives. In the next period $\left(10^{-35} \mathrm{sec}\right.$ to $10^{-15} \mathrm{sec}$ and with temperatures $10^{27}$ to $10^{15}$ kelvin), successive collapses of matter waves, mainly leptons, took place, with velocities of collapse equal to the velocity of light; but slightly smaller than those of the first period. From these collapses, the quarks-antiquarks and the nucleons were created and a few minutes later, the atoms' nuclei were also created. During the period of nuclei creation, the Dwarf Primary Black Holes 
may have been created, in a size of a proton and in insignificant mass, from collapse of matter waves of some billion tons.

In the previously mentioned paper [1], the mathematical formula of transformation of a collapsing mass, in connection with the velocity of collapse (respective to the velocity of evasion on its surface) is introduced. The proof of this formula is presented in detail in this paper, as well as the set of principles on which this particular theory is based. The formula of transformation of the collapsing mass is the following:

$$
\mathrm{m}^{\prime}=\frac{1}{\sqrt{1-\frac{\mathrm{v}_{\infty}^{4}}{\mathrm{c}^{4}}}}\left(\mathrm{~m}-\frac{\mathrm{v}_{\infty}^{2} \mathrm{r}}{2 \mathrm{G}}\right)
$$

and

$$
\mathrm{r}^{\prime}=\frac{\mathrm{r}-2 \mathrm{G} \frac{\mathrm{mv_{ \infty } ^ { 2 }}}{\mathrm{c}^{4}}}{\sqrt{1-\frac{\mathrm{v}_{\infty}^{4}}{\mathrm{c}^{4}}}}
$$

or in table form:

$$
\left[\begin{array}{c}
\mathrm{m}^{\prime} \\
\mathrm{r}^{\prime}
\end{array}\right]=\left[\begin{array}{cc}
\frac{1}{\sqrt{1-\frac{\mathrm{v}_{\infty}^{4}}{\mathrm{c}^{4}}}} & \frac{\frac{\mathrm{v}_{\infty}^{2}}{2 \mathrm{G}}}{\sqrt{1-\frac{\mathrm{v}_{\infty}^{4}}{\mathrm{c}^{4}}}} \\
-\frac{2 \mathrm{G} \frac{\mathrm{v}_{\infty}^{2}}{\mathrm{c}^{4}}}{\sqrt{1-\frac{\mathrm{v}_{\infty}^{4}}{\mathrm{c}^{4}}}} & \frac{1}{\sqrt{1-\frac{\mathrm{v}_{\infty}^{4}}{\mathrm{c}^{4}}}}
\end{array}\right] \times\left[\begin{array}{c}
\mathrm{m} \\
\mathrm{r}
\end{array}\right]
$$

where $\mathrm{m}^{\prime}$ and $\mathrm{r}^{\prime}$ are the collapsing (collapsed) mass and the radius of its volume; $m$ and $r$ are the initial mass and the radius of its critical volume; $\mathrm{v}_{\infty}$ and $\mathrm{c}$ are the velocity of evasion on the surface of the collapsing mass and the velocity of light respectively; and $\mathrm{G}$ is the constant of global traction.

The reverse formula of transformation is the following:

$$
\mathrm{m}=\frac{1}{\sqrt{1-\frac{\mathrm{v}_{\infty}^{4}}{\mathrm{c}^{4}}}}\left(\mathrm{~m}^{\prime}+\frac{\mathrm{v}_{\infty}^{2} \mathrm{r}^{\prime}}{2 \mathrm{G}}\right)
$$

and

$$
r=\frac{r^{\prime}+2 G \frac{m^{\prime} v_{\infty}^{2}}{c^{4}}}{\sqrt{1-\frac{v_{\infty}^{4}}{c^{4}}}}
$$

The reverse formula of transformation (Eq.3) gives the initial mass and the radius of its critical volume. Before the mass gets its critical volume, the velocity of collapse (velocity of evasion by substitution) increases rapidly, while from this volume onwards, the velocity of collapse increases steeply, almost instantly, and as a consequence the gravitational field of the mass increases steeply. This means that, almost till the radius of the critical volume, the gravitational field of the collapsing mass increases rapidly and from this volume onwards, the gravitational field increases steeply, reaching or even exceeding, in intensity, the nuclear field. The verification of the above can be seen through the following examples:

\section{$1^{\text {st }}$ Example:}

As it is known [3], the nucleon's mass is: $\mathrm{m}_{\mathrm{p}}{ }_{\mathrm{p}} \approx 1.674 \times 10^{-}$ ${ }^{27} \mathrm{~kg}$ and the radius of its volume is almost: $\mathrm{r}_{\mathrm{p}}^{\prime} \approx 2.660 \times 10^{-15}$ $\mathrm{m},\left(2.660 \times 10^{-15} \mathrm{~m} \leq \mathrm{r}_{\mathrm{p}}^{\prime}<3 \times 10^{-15} \mathrm{~m}\right)$, as it has been proved in another paper of the author [4]. In that paper, it is proved that the velocity of evasion on the surface of the nucleon is bigger than $83 \%$ of the velocity of light $\left(\mathrm{v}_{\infty}>2.477 \mathrm{x}\right.$ $10^{8} \mathrm{~m} / \mathrm{sec}$ ). Presupposing that the velocity of evasion on the surface of the nucleon is equal to $2.477 \times 10^{8} \mathrm{~m} / \mathrm{sec}$ and that this velocity represents the final velocity of crash (collapse) of its initial mass, emerges that from the application of the formula (Eq.3), the initial mass of the nucleon was $\mathrm{m}_{\mathrm{p}} \approx$ $1.674 \times 10^{12} \mathrm{~kg}(1,674$ billion tons $)$ and the radius of its critical volume $\mathrm{r}_{\mathrm{pcr}} \approx 3.640 \times 10^{-15} \mathrm{~m}$. If we accept that the initial mass did not change (did not decrease) during its crash, but kept its initial quantity, even when this mass was limited to the volume of the nucleon, then the gravitational potential of this mass in the distance of the radius of the nucleon $\left(\mathrm{r}_{\mathrm{p}}^{\prime} \approx 2.660 \times 10^{-15} \mathrm{~m}\right)$ will be: $\mathrm{V}_{\mathrm{p}}=4.51 \times 10^{16} \mathrm{~J} / \mathrm{kg}$. The respective gravitational potential of the nucleon's mass $\left(\mathrm{m}_{\mathrm{p}}^{\prime} \approx 1.674 \mathrm{x} 10^{-27} \mathrm{~kg}\right)$ on its surface is $\mathrm{V}_{\mathrm{p}}^{\prime}=4.51 \times 10^{-23} \mathrm{~J} / \mathrm{kg}$. The ratio of the two potentials is $\mathrm{V}_{\mathrm{p}}: \mathrm{V}_{\mathrm{p}}^{\prime}=10^{39}: 1$, almost the same as the ratio of the nuclear to the gravitational force [5]. The work of collapse, which equals to the difference of the two gravitational potentials, is stored in the form of nucleon's nuclear potential; that is, the nuclear field is a vertical (sudden) change of the gravitational field. Consequently, the nuclear forces are of the same status as the gravitational ones and come from the gravitational potential of the nucleon's initial mass, which is stored in the nucleon.

\section{$2^{\text {nd }}$ Example:}

As it is known [3], the electron's mass is: $\mathrm{m}_{\mathrm{e}}^{\prime} \approx 9.11 \times 10^{-}$ ${ }^{31} \mathrm{~kg}$ and its volume's radius is $\left.\mathrm{r}_{\mathrm{e}}^{\prime} \approx 1.448 \times 10^{-18} \mathrm{~m}_{\left(\mathrm{r}_{\mathrm{e}}^{\prime}\right.}=\mathrm{r}_{\mathrm{p}}^{\prime} / 1837\right)$. If we accept that the final velocity of the electron's initial mass collapse was about $2.993 \times 10^{8} \mathrm{~m} / \mathrm{sec}(0.9983 \mathrm{c})$ and if we suppose that this velocity is equal to the velocity of evasion on the surface of the electron: $\mathrm{v}_{\mathrm{e} \infty} \approx 2.993 \times 10^{8} \mathrm{~m} / \mathrm{sec}$, then from the application of the formula (Eq.3), emerges that the electron's initial mass was $\mathrm{m}_{\mathrm{e}} \approx 9.11 \times 10^{9} \mathrm{~kg}$ (9.11 billion tons) and the radius of the critical volume of this mass $\mathrm{r}_{\mathrm{ecr}} \approx 17.737 \times 10^{-18}$ $\mathrm{m}$. However, if we accept that the initial mass did not change (did not decrease) during its collapse, but kept its initial quantity, even when this mass was limited in the volume of the electron, then the gravitational potential of this mass in the distance of the electron's radius $\left(\mathrm{r}^{\prime} \approx 1.448 \times 10^{-18} \mathrm{~m}\right)$ is $\mathrm{V}_{\mathrm{e}}=41.96 \times 10^{16} \mathrm{~J} / \mathrm{kg}$. The respective gravitational potential of the electron's mass $\left(\mathrm{m}_{\mathrm{e}}^{\prime} \approx 9.11 \times 10^{-31} \mathrm{~kg}\right)$ on its surface is $\mathrm{V}_{\mathrm{e}}^{\prime}=41.96 \times 10^{-}$ 
${ }^{24} \mathrm{~J} / \mathrm{kg}$. The ratio of the two potentials is $\mathrm{V}_{\mathrm{e}}: \mathrm{V}_{\mathrm{e}}^{\prime}=10^{40}: 1$, that is ten times bigger than the ratio of the nuclear to the gravitational force, which means that the electron creates nuclear field too and in fact, at least ten times more powerful than the one of the proton (neutron), but also with a range approximately 266 times smaller.

The radius of gravity of a particle's initial mass is given by the Schwarzschild formula [6]: $r_{p s}=\frac{2 \mathrm{Gm}_{\mathrm{p}}}{\mathrm{c}^{2}}$, where the mass $m_{p}$ is defined by the first relation of the formula (Eq.3). The radius of the critical volume $r_{p c r}$ of the same mass is defined by the second relation of the formula (Eq.3). From the comparison of the two radii, emerges: $r_{p s} \leq r_{p c r}$. It is proved that the two radii become the same $\left(r_{p s}=r_{p c r}\right)$, when the radius of the particle $r_{p}^{\prime}$ coincides with the radius of gravity of the particle's mass, that is:

$$
\mathrm{r}_{\mathrm{p}}^{\prime}=\mathrm{r}_{\mathrm{ps}}^{\prime}=\frac{2 \mathrm{Gm}_{\mathrm{p}}^{\prime}}{\mathrm{c}^{2}}
$$

The relation (Eq.4) shows that the smaller the particle the more these two radii tend to coincide. For instance, the radius of the electron's critical volume has been found in the $2^{\text {nd }}$ Example: $r_{\text {ecr }}=17.737 \times 10^{-18} \mathrm{~m}$. The radius of gravity of the electron's initial mass $\left(\mathrm{m}_{\mathrm{e}}=9.11 \times 109 \mathrm{Kg}\right)$ is $\mathrm{r}_{\mathrm{es}}=13.525 \times 10^{-18} \mathrm{~m}$, i.e. the two radii differ very little. Moreover, the radius of gravity of the nucleon's initial mass is $r_{\mathrm{ps}}=2.484 \times 10^{-15} \mathrm{~m}$, slightly smaller than its radius $\mathrm{r}_{\mathrm{p}}^{\prime}=2.660 \times 10^{-15} \mathrm{~m}$.

From the combination of the formulas (Eq.1) and (Eq.3), the ratio of the collapsing (collapsed) mass $\mathrm{m}^{\prime}$ to the mass $\mathrm{m}$ is given in the radius of its critical volume:

$$
\frac{m^{\prime}}{m}=-\frac{\sqrt{1-\frac{v_{\infty}^{4}}{c^{4}}} r-r^{\prime}}{\sqrt{1-\frac{v_{\infty}^{4}}{c^{4}}} r^{\prime}-r}
$$

The relation (Eq.5) shows that the collapsing mass is not zeroed when the final velocity of collapse (velocity of evasion) becomes equal to the velocity of light and also, in this velocity the collapsing mass gets a negative sign, i.e. it turns into antimass (antimatter). From the formulas (Eq.1), (Eq.3) and (Eq.5), emerges that, for velocities $\mathrm{v}_{\infty}<\mathrm{c}$, we always have:

$$
\sqrt{1-\frac{v_{\infty}^{4}}{c^{4}}} r>r^{\prime}
$$

and

$$
\sqrt{1-\frac{v_{\infty}^{4}}{c^{4}}} r^{\prime}<r
$$

\subsection{The nature and origin of the (elementary) electric charge}

As it is known [3], the electron of the Hydrogen's atom, in its primary state, has a radius of rotation around the atom's nucleus: $r_{1}=0.5293 \times 10^{-10} \mathrm{~m}$ and velocity of rotation $\mathrm{v}_{1}=2.2$ $\mathrm{x} 10^{6} \mathrm{~m} / \mathrm{sec}$. The angular velocity of the electron's rotation around the nucleus emerges from the equation:

$\mathrm{r}_{1} \omega_{1}=\mathrm{v}_{1} \Rightarrow \frac{\mathrm{v}_{1}}{\mathrm{r}_{1}}=\omega_{1}=\frac{2.2 \times 10^{6} \mathrm{~m} / \mathrm{sec}}{0.5293 \times 10^{-10} \mathrm{~m}}=4.157218 \times 10^{16} \mathrm{rad} / \mathrm{sec}$

We know [7] that the impetus of a homogeneous and solid spherical mass $\mathrm{m}$, with mass center $\mathrm{O}$ and radius $\mathrm{r}$, is:

$\mathrm{J}=\frac{2}{5} \mathrm{mr}^{2}$

and its angular momentum is:

$$
\mathrm{L}=\frac{2}{5} \mathrm{mr}^{2} \omega
$$

where $\omega$ is the angular velocity of the rotation of the mass $m$ around an axis which goes through the center of its mass $\mathrm{O}$. The kinetic energy, because of its rotation, is:

$$
\mathrm{E}_{\mathrm{rot}}=\frac{1}{2} \times \frac{2}{5} \mathrm{mr}^{2} \omega^{2}
$$

If the electron is supposed to be a homogeneous and solid spherical mass: $\mathrm{m}_{\mathrm{e}}^{\prime} \approx 9.11 \times 10^{-31} \mathrm{~kg}$, with radius: $\mathrm{r}_{\mathrm{e}}^{\prime} \approx 1.448 \times 10^{-18} \mathrm{~m}$, then its angular momentum from rotation will be:

$$
\mathrm{L}_{\mathrm{e}}^{\prime}=\frac{2}{5} \mathrm{~m}_{\mathrm{e}}^{\prime} \mathrm{r}_{\mathrm{e}}^{\prime 2} \omega_{\mathrm{e}}
$$

where $\omega_{\mathrm{e}}$ is the angular velocity of the electron's rotation around an axis which goes through the centre of its mass. However, the electron has angular momentum (spin) [3]: $\mathrm{s}=\frac{1}{2} \mathrm{~h}\left(\mathrm{~s}=\frac{1}{2} \times \frac{6.626 \times 10^{-34}}{2 \mathrm{p}} \mathrm{Joule} \times \mathrm{sec}\right.$ and for one period $\mathrm{s}=\frac{1}{2} \times \frac{6.626 \times 10^{-34}}{\omega_{\mathrm{e}}}$ Joule ). Consequently, we have the equation:

$$
\frac{2}{5} \mathrm{~m}_{\mathrm{e}}^{\prime} \mathrm{r}_{\mathrm{e}}^{\prime 2} \omega_{\mathrm{e}}^{2}=\frac{1}{2} 6.626 \times 10^{-34} \text { Joule }
$$

From the Equation 11 emerges that:

$$
\omega_{\mathrm{e}}=2.0823 \times 10^{16} \mathrm{rad} / \mathrm{sec}
$$

i.e. the angular velocity of the electron's rotation is about half the angular velocity of the electron's rotation of the Hydrogen's atom, in the radius of its primary state.

The torque $\overline{\mathrm{M}}$ of the external forces is given by the relation [7]: $\overline{\mathrm{M}}=\frac{\mathrm{d} \overline{\mathrm{L}}}{\mathrm{dt}} \quad$ or $\quad \mathrm{M}=\frac{\mathrm{d}}{\mathrm{dt}}\left(\frac{2}{5} \mathrm{mr}^{2} \omega\right)$ and for unchangeable mass $\mathrm{m}$ and stable angular velocity $\omega$ (circular movement) will be: 


$$
\begin{aligned}
& M=\frac{2}{5} \mathrm{~m} \frac{\mathrm{d}}{\mathrm{dt}}\left(\mathrm{r}^{2} \omega\right) \Rightarrow M=\frac{2}{5} \mathrm{~m} \frac{\mathrm{d}}{\mathrm{dt}}(\mathrm{rv})=\frac{2}{5} \mathrm{~m}\left(\frac{\mathrm{vdr}}{\mathrm{dt}}+\frac{\mathrm{rdv}}{\mathrm{dt}}\right)= \\
& \frac{2}{5} \mathrm{~m}\left(\mathrm{v}^{2}+\mathrm{r}^{2} \omega^{2}\right) \Rightarrow M=\frac{4}{5} \mathrm{mr}^{2} \omega^{2}
\end{aligned}
$$

But : $\quad \frac{\mathrm{d} \overline{\mathrm{L}}}{\mathrm{dt}}=\overline{\mathrm{r}} \times \overline{\mathrm{F}} \quad$ and because we have circular

movement :

$$
\frac{\mathrm{dL}}{\mathrm{dt}}=\mathrm{F} \times \mathrm{r}
$$

We observe that the forces' torque is the work of these forces and as a matter of fact, this work is four times the energy from rotation, as it is obvious from the comparison of relations (Eq.9) and (Eq.13). We calculate the work (forces' torque) of the initial mass of the electron $\left(\mathrm{m}_{\mathrm{e}}=9.11 \times 10^{9} \mathrm{~kg}\right)$, with the radius of its critical volume being the radius of rotation and the angular velocity of the electron's rotation being the angular velocity of rotation $\left(\omega_{\mathrm{e}}=2.0823 \times 10^{16} \mathrm{rad} / \mathrm{sec}\right)$, supposing that the electron's initial mass did not decrease during its crash and the angular velocity of rotation of the mass was not different from the one that it possibly had in its critical volume. Thus, we have:

$$
\mathrm{M}_{\text {ecr }}=\frac{4}{5} \times 9.11 \times 10^{9} \times\left(17.737 \times 10^{-18}\right)^{2} \times\left(2.0823 \times 10^{16}\right)^{2}
$$$$
\approx 9.942 \times 10^{8} \text { Joule }
$$

Supposing that this work has been stored in the form of Coulomb potential in the electron, we find this potential in the distance of the electron's radius [8]. So, we have:

$$
\mathrm{V}_{\mathrm{C}}=\frac{\mathrm{K}_{\mathrm{C}} \mathrm{e}}{\mathrm{r}_{\mathrm{e}}^{\prime}}=\frac{8.99 \times 10^{9} \times 1.602 \times 10^{-19}}{1.448 \times 10^{-18}}
$$

\section{$\approx 9.946 \times 10^{8}$ Joule/Coulomb}

We find that the results of the applications (Eq. 15) and (Eq. 16) are almost the same. This discovery verifies the previously mentioned idea, i.e. the work of the forces' torque of the initial mass of the electron has been stored in the form of Coulomb potential, from which comes the Coulomb field of the electron.

This case is the same as the case of the nuclear forces of the nucleon and the electron, which, as mentioned in section 1.2 , comes from the gravitational potential of the nucleon's and electron's initial mass which is stored in the nucleon and electron respectively. We have already found one of the two things we are looking for, that constitute the elementary electric charge, i.e. the one which refers to the origin of the electron's Coulomb field. In order to find the second thing we are looking for, which refers to the "mechanic equivalent" of the electron's electric charge, we obviously have to compare same or similar mechanic or electric quantities, such as the angular momentum from rotation of the electron's initial mass to the energy of the electron's Coulomb field.

The angular momentum from rotation of the initial mass $\left(\mathrm{m}_{\mathrm{e}}\right)$ of the electron, with radius of rotation and angular velocity of rotation those of the electron $\left(\mathrm{r}_{\mathrm{e}}^{\prime}, \omega_{\mathrm{e}}\right)$, is given by the relation:

$$
\mathrm{L}_{\mathrm{e}}=\frac{2}{5} \mathrm{~m}_{\mathrm{e}} \mathrm{r}_{\mathrm{e}}^{\prime 2} \omega_{\mathrm{e}}=\frac{2}{5} \mathrm{~m}_{\mathrm{e}} \mathrm{r}_{\mathrm{e}}^{\prime 2} \frac{\omega_{\mathrm{e}}^{2}}{\omega_{\mathrm{e}}}
$$

and the result of the application of the relation (Eq.17) is :

$\mathrm{L}_{\mathrm{e}}=\frac{2}{5} \times 9.11 \times 10^{9} \times\left(1.448 \times 10^{-18}\right)^{2} \times 2.0823 \times 10^{16}$

$\approx 15.910 \times 10^{-11}$ Joule $\times \mathrm{sec}$

The energy of Coulomb field of the electron is:

$$
\mathrm{U}_{\mathrm{C}}=\frac{\mathrm{k}_{\mathrm{C}} \mathrm{e}^{2}}{\mathrm{r}_{\mathrm{e}}^{\prime}}
$$

and the result of the application of the relation (Eq.19) is:

$$
\mathrm{U}_{\mathrm{C}}=\frac{8.99 \times 10^{9} \times\left(1.602 \times 10^{-19}\right)^{2}}{1.448 \times 10^{-18}}
$$

$$
\approx 15.933 \times 10^{-11} \text { Joule }
$$

We observe that the results of the applications of relations (Eq. 18) and (Eq. 20) are almost the same.

This fact leads us to the thought that the angular momentum from rotation of the electron's initial mass may have been stored in the form of Coulomb field energy.

From the ratio of the relations (Eq. 19) and (Eq. 17) we have:

$$
\begin{aligned}
& \mathrm{e}^{2}=0,4 \frac{\mathrm{m}_{\mathrm{e}}}{\mathrm{K}_{\mathrm{C}}} \mathrm{r}_{\mathrm{e}}^{\prime 3} \omega_{\mathrm{e}} \Rightarrow \mathrm{e} \approx \sqrt{1,6 \pi \varepsilon_{\mathrm{o}} \mathrm{m}_{\mathrm{e}} \mathrm{r}_{\mathrm{e}}^{\prime} \omega_{\mathrm{e}}} \mathrm{r}_{\mathrm{e}}^{\prime} \Rightarrow \\
& \mathrm{e} \approx 1,265 \sqrt{\pi \varepsilon_{\mathrm{o}} \mathrm{m}_{\mathrm{e}} \mathrm{v}_{\text {eot }}^{\prime}} \mathrm{r}_{\mathrm{e}}^{\prime}
\end{aligned}
$$

where $\varepsilon_{\mathrm{o}}$ is the dielectric constant of vacuum.

The relation (Eq. 21) gives the "mechanic equivalent" of the electron's electric charge.

\section{A View about the Possible Size of the Photon}

Today it is scientifically proved that light is twodimensional, that is, on the one hand it is electromagnetic wave, and on the other hand it is particle. Its wavy status is expressed in the phenomena of confluence, diffraction, polarization etc., while its particle status is expressed in the phenomena of interaction of light-matter (absorptionemission), like in the photoelectric phenomenon, in the Copton phenomenon, in the split of nuclei etc. It is also known $[9,10]$ that in big wave lengths, it appears almost exclusively in its wavy status, whereas in small wave lengths (ultra-violet and others), its particle status prevails. Since the light is also a particle, it should logically have a size (volume). The smaller the wave length $\lambda$ gets, the more it will tend to acquire particle's size, and in particular the $\lambda / 2$ will lean towards the particle's radius. Let's try such an approach in the size of the electron. We previously supposed that the radius of the electron's volume is approximately $\mathrm{r}_{\mathrm{e}} \approx 1.448 \times 10^{-18} \mathrm{~m}$. The electron, however, as a particle, has also a wavy status (it is a wave), the length of which, according to the De Broglie theory, is given by the relation: 
$\lambda_{\mathrm{e}}=\frac{\mathrm{h}}{\mathrm{P}_{\mathrm{e}}}$, where $\mathrm{h}$ is the Planck constant and $\mathrm{P}_{\mathrm{e}}$ is the electron's momentum.

We suppose that an electron has kinetic energy:

$\mathrm{E}_{\mathrm{e}}=1 \mathrm{eV}=1.602 \times 10^{-19}$ Joule

The momentum $\mathrm{P}_{\mathrm{e}}$ is given by the formula:

$\mathrm{P}_{\mathrm{e}}=\sqrt{2 \mathrm{~m}_{\mathrm{e}} \mathrm{E}_{\mathrm{e}}}$

where $\mathrm{m}_{\mathrm{e}}$ is the tranquillity mass of the electron $\left(\mathrm{m}_{\mathrm{e}}=9.11 \mathrm{x}\right.$ $10^{-31} \mathrm{~kg}$ ). So, the wave length will be:

$$
\lambda_{\mathrm{e}}=\frac{\mathrm{h}}{\sqrt{2 \mathrm{~m}_{\mathrm{e}} \mathrm{E}_{\mathrm{e}}}}
$$

From the relation (Eq. 23) emerges that:

$$
\begin{aligned}
& \lambda_{\mathrm{e}}=1.22644 \times 10^{-9} \mathrm{~m} \quad \text { and } \\
& \frac{\lambda_{\mathrm{e}}}{2} \approx 6.1322 \times 10^{-10} \mathrm{~m} .
\end{aligned}
$$

In the big Stanford's linear accelerator of particles, $3.2 \mathrm{~km}$ long, the following electrons' energy has been achieved: $\mathrm{E} \approx$ $20 \mathrm{GeV}$, which corresponds to electrons' velocity: $\mathrm{v}_{\mathrm{e}}=$ $0.9999999996 \mathrm{c}$. The electron's momentum in this case is given by the relativistic formula:

$$
P_{e} c=\sqrt{E^{2}-E_{o}^{2}}
$$

where $E_{o}$ is the tranquillity energy of the electron $\left(E_{0} \approx 0.511\right.$ $\mathrm{MeV})$ and $\mathrm{c}$ is the velocity of light $\left(\mathrm{c}=2.998 \times 10^{8} \mathrm{~m} / \mathrm{sec}\right)$. So, the wave length will be: $\lambda_{\mathrm{e}}=\frac{\mathrm{h}}{\mathrm{P}_{\mathrm{e}}} \approx 6.183 \times 10^{-17} \mathrm{~m}$ and $\frac{\lambda_{\mathrm{e}}}{2} \approx 3.09 \times 10^{-17} \mathrm{~m}$. We observe that the $\lambda_{\mathrm{e}} / \mathbf{2}$ approaches the electron's radius $\left(\mathrm{r}_{\mathrm{e}} \approx 1.448 \times 10^{-18} \mathrm{~m}\right)$.

We will now try a similar approach with photons of high energy. We know that some photons of radiation $\gamma$, which have been found in the highest levels of atmosphere (having been produced by the reaction of cosmic rays with air atoms) have $E_{\mathrm{ph}}$ energies that even reach $10^{14} \mathrm{eV}$. The relation that gives the momentum $\mathrm{P}_{\mathrm{ph}}$ of these photons is:

$$
\mathrm{P}_{\mathrm{ph}} \mathrm{c}=\mathrm{E}_{\mathrm{ph}}
$$

And the relation that gives the wave length $\lambda_{\mathrm{ph}}$ is:

$$
\lambda_{\mathrm{ph}}=\frac{\mathrm{h}}{\mathrm{P}_{\mathrm{ph}}}=\frac{\mathrm{hc}}{\mathrm{E}_{\mathrm{ph}}}
$$

The relation (Eq. 26) gives a value of wave length: $\lambda_{\mathrm{ph}} \approx 1.24 \times 10^{-20} \mathrm{~m}$ and $\frac{\lambda_{\mathrm{ph}}}{2} \approx 6.2 \times 10^{-21} \mathrm{~m}$

If we suppose that the following relation is valid:

$\frac{\mathrm{r}_{\mathrm{ph}}}{\lambda_{\mathrm{ph}} / 2} \leq \frac{\mathrm{r}_{\mathrm{e}}}{\lambda_{\mathrm{e}} / 2}$

where $r_{p h}$ is the possible radius of the photon's particle, then from the relation (Eq. 28) emerges that:

$\frac{\mathrm{r}_{\mathrm{ph}}}{6.2 \times 10^{-21}} \leq \frac{1.448 \times 10^{-18}}{3.09 \times 10^{-17}} \Rightarrow \mathrm{r}_{\mathrm{ph}} \leq 2.9 \times 10^{-22} \mathrm{~m}$

If we compare the radii $r_{p h} \kappa \alpha \iota r_{e}$, we have the following result: $r_{p h} \leq 2 \times 10^{-4} r_{e}$, which shows that the possible radius of the particle of this particular radiation is by 20,000 times smaller than that of the electron. If we accept the theory of Dr. S. Hawking [2], about the creation of the Universe, where he assumes that the absolute temperature ( $\mathrm{T}$ ${ }^{\circ} \mathrm{K}$ ) of the Universe in the period (season) of the Big Unification was $\mathrm{T}=10^{32}$ kelvin, then according to the Wien law, the radiation of this period would have a wave length:

$\lambda_{\mathrm{ph}}=\frac{\mathrm{hc}}{4.965 \mathrm{kT}}$

where $\mathrm{h}$ is the Planck constant, $\mathrm{c}$ is the velocity of light and $\mathrm{k}$ is the Boltzmann constant $\left(\mathrm{k}=1.381 \times 10^{-23} \mathrm{Joule} /{ }^{0} \mathrm{~K}\right)$.

The formula (Eq. 30) gives a wave length:

$\lambda_{\mathrm{ph}} \approx 2.897 \times 10^{-35} \mathrm{~m}$ and $\frac{\lambda_{\mathrm{ph}}}{2} \approx 1.45 \times 10^{-35} \mathrm{~m}$

If we suppose that the relation (Eq. 28) is valid, then the possible particle's radius of the radiation of this period will be:

$\mathrm{r}_{\mathrm{ph}} \leq 6.8 \times 10^{-37} \mathrm{~m}$

However, the length of this radius, could be considered unrealistic, because it is smaller than the Planck length, which is supposed to be, in quantum mechanics, the lowest level of wave length, the detection of which is theoretically possible. The Planck length is given by the relation:

$\mathrm{I}_{\mathrm{pl}}=\sqrt{\mathrm{r}_{\mathrm{q}} \times \mathrm{r}_{\mathrm{s}}}=\sqrt{\frac{\mathrm{h}}{2 \mathrm{mc}} \times \frac{2 \mathrm{Gm}}{\mathrm{c}^{2}}}=\sqrt{\frac{\mathrm{Gh}}{\mathrm{c}^{3}}} \approx 4.05 \times 10^{-35} \mathrm{~m}$

The factor $\mathrm{r}_{\mathrm{q}}=\frac{\mathrm{h}}{2 \mathrm{mc}}$ is called quantum radius of mass $\mathrm{m}$ and defines a wave length $(\boldsymbol{\lambda} / \mathbf{2})$ according to De Broglie, while the factor $r_{s}=\frac{2 \mathrm{Gm}}{\mathrm{c}^{2}}$ is the gravity radius (Schwarrzshild radius) of the same mass. These two factors, 
although they concern the same material body (the same tranquillity mass), refer to different states of this body. That is because, the quantum radius refers to a supposedly moving material body, the momentum of which is the product of its mass multiplied by the velocity of light while the gravity radius refers to the horizon of events of the same body, if we suppose that its mass is so shrunk that it can be included in the volume of this radius. Adopting this view, we could downsize, theoretically, the Planck length, by rewriting the relation (Eq.33), in the form:

$\mathrm{I}_{\mathrm{pl}}=\sqrt{\frac{\mathrm{h} \sqrt{1-\mathrm{v}^{2} / \mathrm{c}^{2}}}{2 \mathrm{~m}_{\mathrm{o}} \mathrm{c}} \times \frac{2 \mathrm{Gm}_{\mathrm{o}}}{\mathrm{c}^{2}}}=\sqrt[4]{1-\frac{\mathrm{v}^{2}}{\mathrm{c}^{2}}} \times \sqrt{\frac{\mathrm{Gh}}{\mathrm{c}^{3}}}$

For the case of the moving electron with velocity $\mathrm{v}_{\mathrm{e}}=$ $0.9999999996 \mathrm{c}$, whose wave length is used for the comparison, according to the relation (Eq.34), the Planck length will be:

$\mathrm{I}_{\mathrm{pl}}=\sqrt[4]{1-0.9999999996^{2}} \times \sqrt{\frac{\mathrm{Gh}}{\mathrm{c}^{3}}} \approx 2.2775 \times 10^{-37} \mathrm{~m}$
If we accept the result of the relation (Eq. 35) to be the Planck length, then the length of the photon's radius, which was found by the relation (Eq. 32), seems logical.

\section{Conclusion}

Overall, in this paper, we introduced two issues that we tried and, to a great extent we think we achieved, to address adequately. The one was about the nature and the origin of the electric charge and the other was about the possible size of the photon.

As for electron's electric charge, we found that it is a form of stored angular momentum from rotation of the electron's initial mass and it took place during the Big Bang.

As for the possible size of the photon, we found that the radius of the size of the photon, as a particle is at least 100 times smaller than Plank length.

The fact that we tried for every our assumption to provide a mathematical formula is, in our opinion, of great significance, as it can be of much help to the scientists who are trying to shed light to the mystery of the Universe's creation.

\section{References}

1. Ch. Matsiras, Dwarf Primary Black Holes and Nucleons- Indirect Theory of Unified Fields - Relation between Gravitational and Nuclear potentials, The Physical World, Vol. 22 (181), p. 40-49 (Dec. - Feb. 2006).

2. S. Hawking, A Brief History of Time, Cambridge University Press, Cambridge (1973).

3. St. Charalambous, Introduction to Atomic and Molecular Physics, University Editions, Thessaloniki (1975).

4. Ch. Matsiras, Thoughts and Views about the Atoms' Nuclei, The Physical World, Vol. 24 (184), p. 32-36 (Jun. - Aug. 2006).

5. St. Charalambous, Introduction to Nuclear Physics, University Editions, Thessaloniki (1981).
6. G. Kontopoulos, Introduction to Astrophysics, Vol.4, Cosmology, University Editions, Thessaloniki, (1970).

7. I. Chatzidimitriou, Theoretical Mechanics, Volume II, University Editions, Thessaloniki (1971)

8. M. Schwartz, Principles of Electrodynamics, University Editions, Thessaloniki (1979).

9. M. Grypeos, Quantum Mechanics Lessons, Volume A, University Editions, Thessaloniki (1978)

10. D.C. Heath and Company Lexington, PSSC PHYSICS, $6^{\text {th }}$ Edition, Massachusetts Toronto (1985) 